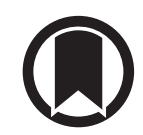

CrossMark

\title{
Video-assisted thoracic lobectomy versus stereotactic body radiotherapy for stage I nonsmall cell lung cancer in elderly patients: a propensity matched comparative analysis
}

\author{
Deniece D.E.M.A. Detillon ${ }^{1,2}$, Mieke J. Aarts ${ }^{3}$, Katrien De Jaeger ${ }^{4}$, \\ Casper H.J. Van Eijck ${ }^{2}$ and Eelco J. Veen ${ }^{1}$
}

Affiliations: ${ }^{1}$ Dept of Surgery, Amphia Hospital, Breda, The Netherlands. ${ }^{2}$ Dept of Surgery, Erasmus Medical Center, Rotterdam, The Netherlands. ${ }^{3}$ Netherlands Cancer Registry, Netherlands Comprehensive Cancer Organization, Utrecht, The Netherlands. "'Dept of Radiotherapy, Catharina Hospital, Eindhoven, The Netherlands.

Correspondence: Deniece D.E.M.A. Detillon, Dept of Surgery, Amphia Hospital, Molengracht 21, Breda, 4818 CK, The Netherlands. E-mail: DDetillondamphia.nl

@ERSpublications

This matched analysis compared VATS lobectomy with SBRT in patients aged $\geqslant 65$ years with clinical stage I NSCLC. VATS lobectomy resulted in better overall survival, which is important in decisionmaking for elderly patients who can tolerate surgery. http://ow.ly/LkWz30o8pXv

Cite this article as: Detillon DDEMA, Aarts MJ, De Jaeger K, et al. Video-assisted thoracic lobectomy versus stereotactic body radiotherapy for stage I nonsmall cell lung cancer in elderly patients: a propensity matched comparative analysis. Eur Respir J 2019; 53: 1801561 [https://doi.org/10.1183/13993003.015612018].

ABSTRACT Comparative studies of the overall survival (OS) in elderly patients with nonsmall cell lung cancer (NSCLC) after surgery or stereotactic body radiotherapy (SBRT) have been limited by mixed extents of resection and different surgical approaches.

792 patients aged $\geqslant 65$ years with clinical stage I NSCLC underwent video-assisted thoracic surgery (VATS) lobectomy or SBRT between 2010 and 2015. The propensity score-matched primary analysis included data from the full cohort; the secondary analysis included data from a subgroup of patients with data on pulmonary function.

Median OS for unmatched patients was 77 months for patients undergoing VATS lobectomy and 38 months for SBRT. The 1-, 3- and 5-year OS rates after VATS lobectomy were $92 \%, 76 \%$ and $65 \%$, and after SBRT were 90\%, 52\% and 29\% ( $\mathrm{p}<0.001)$. Median OS for matched patients in the primary analysis was 77 months for patients undergoing VATS lobectomy and 33 months for SBRT. The 1-, 3- and 5-year OS rates after VATS lobectomy were $91 \%, 68 \%$ and 58\%, and after SBRT were $87 \%$, 46\% and 29\% $(\mathrm{p}<0.001)$. The survival advantage with VATS lobectomy persisted in the secondary analysis after adjusting for non-matched variables $(\mathrm{p}=0.034)$.

We suggest that elderly patients with stage I NSCLC undergoing VATS lobectomy have a better rate of OS than patients undergoing SBRT, irrespective of matching. This could be clinically important in decision-making for elderly patients who can tolerate surgery. 


\section{Introduction}

Lung cancer is the leading cause of cancer death worldwide and in the Netherlands [1]. The highest incidence rates are found among men and women aged 70-79 years [1]. On the basis of demographic developments, the absolute number of new cases of lung cancer in the Netherlands is expected to increase by $37 \%$ between 2015 and 2040 [2].

Surgery offers the best potential cure for early-stage nonsmall cell lung cancer (NSCLC). It is not always offered, however, to elderly patients in view of their advanced age and comorbidities. In our previous study, only $52 \%$ of 2168 patients aged $\geqslant 65$ years with stage I NSCLC were treated surgically and $15 \%$ did not receive any cancer-directed therapy [3]. Video-assisted thoracic surgery (VATS) is a minimally invasive surgical approach that was introduced in the Netherlands in 2006 and has been found superior to thoracotomy in terms of post-operative morbidity [4-6].

For patients with inoperable disease, radiotherapy is an alternative treatment with curative intent $[6,7]$. Stereotactic body radiotherapy (SBRT) became available in the Netherlands in 2003 and became widespread after 2007 [6]. SBRT consists of the delivery of high doses of radiation in a limited number of fractions. SBRT is superior to conventionally fractionated radiotherapy in terms of local control and overall survival (OS) [8].

Studies comparing surgery with SBRT have produced conflicting OS results. Most of these studies have been limited by different surgical approaches (thoracotomy and VATS) [9-17] and mixed extents of resection (sublobar, lobectomy, bilobectomy, pneumonectomy) [10, 13, 16-18]. Therefore, we performed a study comparing OS between VATS lobectomy and SBRT for patients aged $\geqslant 65$ years with clinical stage I NSCLC, taking into account differences in patient and tumour characteristics by using propensity score matching.

\section{Materials and methods}

Population-based data from the southern region of the Netherlands Cancer Registry (NCR) were used, because information on comorbidities is routinely collected only in this region. This region encompasses 2.4 million inhabitants (15\% of the Dutch population). The NCR records data from all patients diagnosed with cancer and receives notifications of all newly diagnosed malignancies from the nationwide network and registry of histopathology and cytopathology in the Netherlands. Additional sources of data are radiotherapy institutes and the Dutch national registry of hospital discharge. These data are supplemented with data from medical records. Cause of death was not available. Follow-up data were complete until February 2018.

We retrieved data for all patients aged $\geqslant 65$ years diagnosed between 2010 and 2015 with clinical stage I NSCLC, according to the International Union Against Cancer Tumour Node Metastasis (TNM) edition 7 [19], whose primary treatment was VATS lobectomy or SBRT. The VATS procedure involved a multiportal approach without rib spreading. SBRT schedules varied between three to eight fractions delivered two to three times per week in case of multiple fractions. The NCR records the primary treatment given; it was not always clear if this was the same treatment as the intended treatment for surgical patients. The NCR does record VATS conversions, which resulted in some patients having a different extent of resection than

\section{TABLE 1 Adapted version of Charlson Comorbidity Index}

\begin{tabular}{ll} 
Score & Condition \\
\hline 1 & Cardiac disease \\
& Vascular disease \\
& Hypertension \\
& Cerebrovascular disease \\
& Dementia \\
& Chronic pulmonary disease \\
& Connective tissue disease \\
& Digestive tract disease \\
& Liver disease \\
& Diabetes mellitus \\
& Auto-immune disease \\
& Kidney disease \\
& Previous malignancy \\
& Infectious disease \\
&
\end{tabular}


lobectomy. An intention-to-treat analysis was performed, i.e. when a conversion was recorded with a resection other than lobectomy, the patient was analysed as having undergone VATS lobectomy (from now on referred to as intended VATS lobectomy). Demographic variables retrieved were age and sex. A slightly adapted version of the Charlson Comorbidity Index (CCI) was used (table 1). Comorbidities were

TABLE 2 Characteristics of unmatched patients aged $\geqslant 65$ years undergoing intended VATS lobectomy or SBRT for clinical stage I nonsmall cell lung cancer

\begin{tabular}{|c|c|c|c|c|}
\hline Characteristics & VATS & SBRT & Standardised difference & p-value \\
\hline Subjects $\mathrm{n}$ & 414 & 378 & & \\
\hline Age years & $72.2 \pm 4.8$ & $74.9 \pm 5.9$ & -0.50 & $<0.001$ \\
\hline Sex & & & 0.05 & 0.467 \\
\hline Male & $256(61.8)$ & 224 (59.3) & & \\
\hline Female & $158(38.2)$ & $154(40.7)$ & & \\
\hline FEV $1 \%$ pred ${ }^{\#}$ & $88.5 \pm 18.8$ & $67.6 \pm 24.8$ & 0.92 & $<0.001$ \\
\hline D.co \% pred ${ }^{\#}$ & $87.3 \pm 19.3$ & $69.0 \pm 21.4$ & 0.90 & $<0.001$ \\
\hline \multicolumn{5}{|l|}{ Comorbidities } \\
\hline Pulmonary & $130(34.6)$ & 221 (61.9) & -0.57 & $<0.001$ \\
\hline Cardiac & $133(35.4)$ & $158(44.3)$ & -0.18 & 0.016 \\
\hline Hypertension & $152(40.4)$ & 139 (38.9) & 0.03 & 0.706 \\
\hline Previous malignancy & $106(28.2)$ & $126(35.3)$ & -0.15 & 0.039 \\
\hline Vascular & $101(26.9)$ & $116(32.5)$ & -0.12 & 0.106 \\
\hline Diabetes & 57 (15.2) & $73(20.4)$ & -0.14 & 0.066 \\
\hline Unknown & $38(9.2)$ & $21(5.6)$ & & \\
\hline Number of comorbidities & & & -0.42 & $<0.001$ \\
\hline s & $43(11.4)^{*}$ & $9(2.5)$ & & \\
\hline 1 & $97(25.8)$ & $73(20.5)$ & & \\
\hline 2 & $112(29.8)$ & $105(29.4)$ & & \\
\hline 3 & $68(18.1)$ & 75 (21.0) & & \\
\hline$\geqslant 4$ & 56 (14.9)* & $95(26.6)$ & & \\
\hline $\mathrm{CCl}$ score & & & -0.37 & $<0.001$ \\
\hline 0 & $43(11.4)^{*}$ & $9(2.5)$ & & \\
\hline 1 & $73(19.4)$ & $61(17.1)$ & & \\
\hline 2 & $100(26.6)$ & $79(22.1)$ & & \\
\hline 3 & $70(18.6)$ & 83 (23.2) & & \\
\hline$\geqslant 4$ & 90 (23.9)* & $125(35.0)$ & & \\
\hline \multicolumn{5}{|l|}{ Clinical tumour $\mathrm{T}$ stage } \\
\hline T1a $(\leqslant 2 \mathrm{~cm})$ & $139(33.6)$ & $139(36.8)$ & -0.10 & 0.20 \\
\hline $\mathrm{T} 1 \mathrm{~b}(>2-\leqslant 3 \mathrm{~cm})$ & $111(26.8)$ & 117 (31.0) & -0.12 & 0.11 \\
\hline T2a $(>3-\leqslant 5 \mathrm{~cm})$ & $143(34.5)$ & $91(24.1)$ & 0.22 & 0.003 \\
\hline Unknown & $21(5.1)$ & $31(8.2)$ & & \\
\hline Tumour location & & & 0.02 & 0.667 \\
\hline Right upper lobe & $145(35.0)$ & $129(34.1)$ & & \\
\hline Right middle lobe & $18(4.3)$ & $12(3.2)$ & & \\
\hline Right lower lobe & 64 (15.5) & $59(15.6)$ & & \\
\hline Left upper lobe & $112(27.1)$ & 119 (31.5) & & \\
\hline Left lower lobe & $70(16.9)$ & 58 (15.3) & & \\
\hline Unknown & $5(1.2)$ & $1(0.3)$ & & \\
\hline \multicolumn{5}{|l|}{ Tumour histology } \\
\hline Adenocarcinoma & $198(47.8)$ & $77(20.4)$ & 0.60 & $<0.001$ \\
\hline Squamous cell carcinoma & 142 (34.3) & 65 (17.2) & 0.40 & $<0.001$ \\
\hline Other ${ }^{\text {I }}$ & 72 (17.4) & $50(13.2)$ & 0.12 & 0.115 \\
\hline Unknown & $2(0.5)$ & $186(49.2)$ & -1.39 & $<0.001$ \\
\hline Pathological confirmation & & & 1.38 & $<0.001$ \\
\hline Yes & $414(100)$ & $198(52.4)$ & & \\
\hline No & - & $180(47.6)$ & & \\
\hline
\end{tabular}

Data are presented as $\mathrm{n}(\%)$ or mean $\pm \mathrm{SD}$, unless otherwise stated. VATS: video-assisted thoracic surgery; SBRT: stereotactic body radiotherapy; FEV1 \% pred: percentage predicted forced expiratory volume in $1 \mathrm{~s}_{\text {; }}$ $D\llcorner C 0 \%$ pred: percentage predicted diffusing capacity of the lung for carbon monoxide; CCl: Charlson Comorbidity Index. " : based on patients with available data on pulmonary function ( $n=169)$; ๆ: the 180 patients without pathological confirmation undergoing SBRT are included in "unknown" tumour histology; *: $p \leqslant 0.05$ for comparison of intended VATS lobectomy versus SBRT. 
analysed as number $(0,1,2,3, \geqslant 4)$ and adapted CCI score $(0,1,2,3, \geqslant 4)$. Tumour characteristics included clinical tumour stage, tumour location, histology and the presence or absence of pathological confirmation. Dutch national radiotherapy guidelines indicate that patients without pathological confirmation are eligible for radiotherapy in case of 1) a new or growing lesion on computed tomography (CT) scans with characteristics of malignancy, 2) a high risk for developing lung cancer based on age and smoking history and 3) a fluorodeoxyglucose positron emission tomography (FDG-PET) positive lesion [20]. According to the Dutch practice guidelines, all patients with NSCLC who are eligible for curative treatment should undergo PET-CT [7]. The NCR recorded whether a PET-CT was performed, which turned out to be the case in $75-90 \%$ of patients. Criteria to perform lung surgery were in accordance with the Dutch practice guidelines for the treatment of NSCLC [7]. Pulmonary function is not recorded in the database. Because we wished to include pulmonary function data as well, we identified patients diagnosed in one specific hospital (Amphia Hospital, Breda, the Netherlands), reviewed their charts manually and retrieved results on percentage predicted forced expiratory volume in $1 \mathrm{~s}$ (FEV1 \% pred) and percentage predicted diffusing capacity of lung for carbon monoxide (DLCO \% pred).

\section{Statistical analysis}

Two analyses were performed using propensity score matching: the primary analysis included data from the full cohort and the secondary analysis included data from the subgroup of patients with data on pulmonary function. Propensity score matching reduces treatment selection bias when estimating causal treatment effects using observational data [21]. Patients were matched separately for the primary and secondary analysis using logistic regression, with the dependent variable being intended VATS lobectomy versus SBRT. Independent variables were selected on the basis of previous studies. Those included in the primary analysis were age, sex, all individual comorbidities from the adapted CCI, CCI score, number of comorbidities, clinical tumour $\mathrm{T}$ stage, tumour location, tumour histology and pathological confirmation $[9,10,12,13,15,18,22-25]$. In the secondary analysis, we included $\mathrm{FEV}_{1} \%$ pred $[9,13,23-25]$ and DLCO \% pred [13] as independent variables, as well as all variables from the primary analysis except all individual comorbidities in the adapted CCI, tumour histology and pathological confirmation. These latter variables were excluded because they resulted in just a few matches. In the secondary analysis we made a distinction between the analysis with and without adjustment for an unbalanced matched variable and the unbalanced non-matched variables. In both analyses, patients were matched using one-to-one nearest neighbour matching without replacement. Calipers of a width equal to $0.2 \mathrm{sD}$ of the logit of the propensity score were used. This width results in optimal estimation of risk differences [21]. Balance was assessed with standardised differences because these are not influenced by sample size [26]. Standardised differences for almost all covariates were $<0.1$, indicating adequate balance [27]. The standardised differences for the remaining few covariates were $>0.1$ but $<0.2$, denoting only a small effect on balance [28].

In the full cohort, variables were compared using the Chi-squared test and t-test. In the matched cohorts, variables were compared using the McNemar test and the Wilcoxon signed rank test. Kaplan-Meier survival curves were constructed and p-values were calculated using the logrank test in the full cohort and Cox proportional hazard models stratified by matched pairs in the matched cohorts [29]. Hazard ratios (HR) and 95\% confidence intervals were calculated for the first 15 months of follow-up and after 15 months of follow-up because the Kaplan-Meier curves were quite similar in the first 15 months. All statistical tests were two-sided, and $\mathrm{p} \leqslant 0.05$ was considered to indicate statistical significance. Statistical analyses were performed using SPSS statistical software (SPSS version 22; Chicago, IL, USA).

\section{Results}

Between 2010 and 2015, 625 patients aged $\geqslant 65$ years underwent lobectomy for stage I NSCLC: 211 $(33.8 \%)$ by thoracotomy and $414(66.2 \%)$ were scheduled for VATS. 490 patients were treated with radiotherapy: $112(22.9 \%)$ with conventional radiotherapy and $378(77.1 \%)$ with SBRT. In the present study we focused on 792 patients who underwent intended VATS lobectomy or SBRT. The mean \pm SD age of the studied group was $73.5 \pm 5.5$ years and $62 \%$ were male. Pulmonary comorbidities were documented in 351 patients (44\%), cardiac comorbidities in 291 patients $(37 \%)$ and 232 patients $(29 \%)$ had a history of a previous malignancy. Patients undergoing intended VATS lobectomy were younger $(p<0.001)$, had better pulmonary function $(p<0.001)$ and tended to have lower CCI scores $(p<0.001)$ than patients undergoing SBRT (table 2). Tumour location was similar between groups. The presence of a T2a tumour significantly differed between groups: $35 \%$ of patients undergoing intended VATS lobectomy versus $24 \%$ of patients undergoing SBRT $(\mathrm{p}=0.003)$. VATS conversions were recorded in 68 patients $(16 \%)$. Four patients had a different extent of resection than lobectomy only (one lobectomy and wedge resection, one lobectomy and segmentectomy, one wedge resection, and one lobectomy and rib resection). When table 2 was reconstructed with the exclusion of the 68 patients in whom a conversion was recorded, differences between patients undergoing VATS lobectomy and SBRT remained the same. 
Pathological confirmation of malignancy was not obtained in $48 \%$ of patients undergoing SBRT. Pathological upstaging was documented for 92 patients (22\%) after surgery; cT1-2a became pT2b-4 in 48 patients $(12 \%)$, and $\mathrm{cN} 0$ became $\mathrm{pN} 1$ in 36 patients $(9 \%)$ and $\mathrm{pN} 2$ in 20 patients (5\%). 12 patients (3\%) were upstaged to pT2b-4 and pN1-2. Adjuvant treatment was administered to 48 upstaged patients (52\%): 40 patients received chemotherapy, six radiotherapy and two chemoradiotherapy.

The median follow-up for all patients was 34 months; for patients undergoing intended VATS lobectomy it was 38 months, and for those undergoing SBRT it was 32 months. The estimated median OS time for all patients was 56 months (95\% CI 49-62 months); for patients undergoing intended VATS lobectomy it was 77 months (95\% CI 72 months-not available (NA), the upper limit of the 95\% confidence band around the survival curve did not drop below 0.50), and for patients undergoing SBRT it was 38 months (95\% CI 34-42 months, $\mathrm{p}<0.001)$. The 1-, 3- and 5-year OS rates for patients undergoing intended VATS lobectomy were $92 \%, 76 \%$ and $65 \%$, and for patients undergoing SBRT were $90 \%, 52 \%$ and $29 \%$ (logrank $\mathrm{p}<0.001$, figure 1a). Patients undergoing intended VATS lobectomy had an OS advantage in both the first 15 months (HR 0.66, 95\% CI 0.44-0.99) and after 15 months (HR 0.33, 95\% CI 0.26-0.43, table 3) of follow-up. For patients undergoing intended VATS lobectomy, age was the only clinical characteristic associated with OS after 15 months of follow-up (HR 1.07, 95\% CI 1.02-1.12, p=0.004). The 5-year OS rate was $52 \%$ for upstaged patients receiving adjuvant treatment and $39 \%$ for those not receiving adjuvant treatment. Patients who were not upstaged had a 5 -year OS rate of $70 \%$.

\section{Primary analysis: intended VATS lobectomy versus SBRT for the full cohort of patients}

After matching, the cohort consisted of 159 patients in each treatment arm (table 4). Balance was assessed; differences between patients in both groups were minimal or absent. Interestingly, the most compromised intended VATS lobectomy patients were matched, i.e. those with higher age and greater comorbidity burden and CCI score than unmatched patients.

The median follow-up for all matched patients was 33 months; for patients undergoing intended VATS lobectomy it was 35 months, and for those undergoing SBRT it was 32 months. The estimated median OS for patients undergoing intended VATS was 77 months (95\% CI 59 months-NA, the upper limit of the 95\% confidence band around the survival curve did not drop below 0.50) and for those undergoing SBRT it was 33 months (95\% CI 30-37 months, p<0.001). The 1-, 3- and 5-year OS rates for patients undergoing intended VATS were $91 \%, 68 \%$ and $58 \%$, and for patients undergoing SBRT were $87 \%, 46 \%$ and $29 \%(\mathrm{p}<0.001$, figure $1 \mathrm{~b})$. There was no difference in OS between the groups in the first 15 months of follow-up (HR 0.87, 95\% CI 0.49-1.55). After 15 months, OS significantly favoured intended VATS lobectomy (HR 0.38, 95\% CI 0.26-0.56, table 3).

\section{Secondary analysis: intended VATS lobectomy versus SBRT for the subgroup of patients with data available on pulmonary function}

After matching, the cohort consisted of 36 patients in each treatment arm (table 4). Balance was assessed; tumour stage cT1a and the non-matched variables histology and pathological confirmation were not well balanced (standardised differences ranging from 0.26 to 1.68). Interestingly, less compromised SBRT patients were matched, i.e. those with lower age, better pulmonary function, and a lower comorbidity burden and CCI score than unmatched patients.

The median follow-up for all matched patients was 38 months; for patients undergoing intended VATS lobectomy it was 42 months, and for those undergoing SBRT it was 33 months. The estimated median OS for patients undergoing intended VATS was 77 months (95\% CI 68-86 months) and for those undergoing SBRT was 51 months (95\% CI 26-76 months, $\mathrm{p}=0.087$ ). The 1-, 3- and 5-year OS rates for patients undergoing intended VATS were $92 \%, 72 \%$ and $65 \%$, and those for patients undergoing SBRT were $89 \%$, $63 \%$ and 49\% ( $\mathrm{p}=0.087$; adjusted for $\mathrm{cT} 1 \mathrm{a}$, histology and pathological confirmation $\mathrm{p}=0.034$, figure $1 \mathrm{c}$ ). There was no difference in OS between the groups in the first 15 months of follow-up (HR 1.23, 95\% CI 0.38-4.03; adjusted for cT1a, histology and pathological confirmation HR 0.67, 95\% CI 0.17-2.69). After 15 months, OS significantly favoured intended VATS lobectomy (HR 0.36, 95\% CI 0.15-0.86; adjusted for cT1a, histology and pathological confirmation HR 0.30, 95\% CI 0.11-0.81; table 3).

\section{Discussion}

This study demonstrates survival outcomes for patients aged $\geqslant 65$ years with clinical stage I NSCLC diagnosed between 2010 and 2015 and treated with intended VATS lobectomy or SBRT. In unmatched and propensity score-matched primary analysis, median OS as well as 1-, 3- and 5-year OS was significantly better for those treated with intended VATS lobectomy than for those treated with SBRT. In the secondary analysis, in which pulmonary function was taken into account, the survival advantage related to intended VATS lobectomy persisted after adjusting for non-matched variables. This is in line 

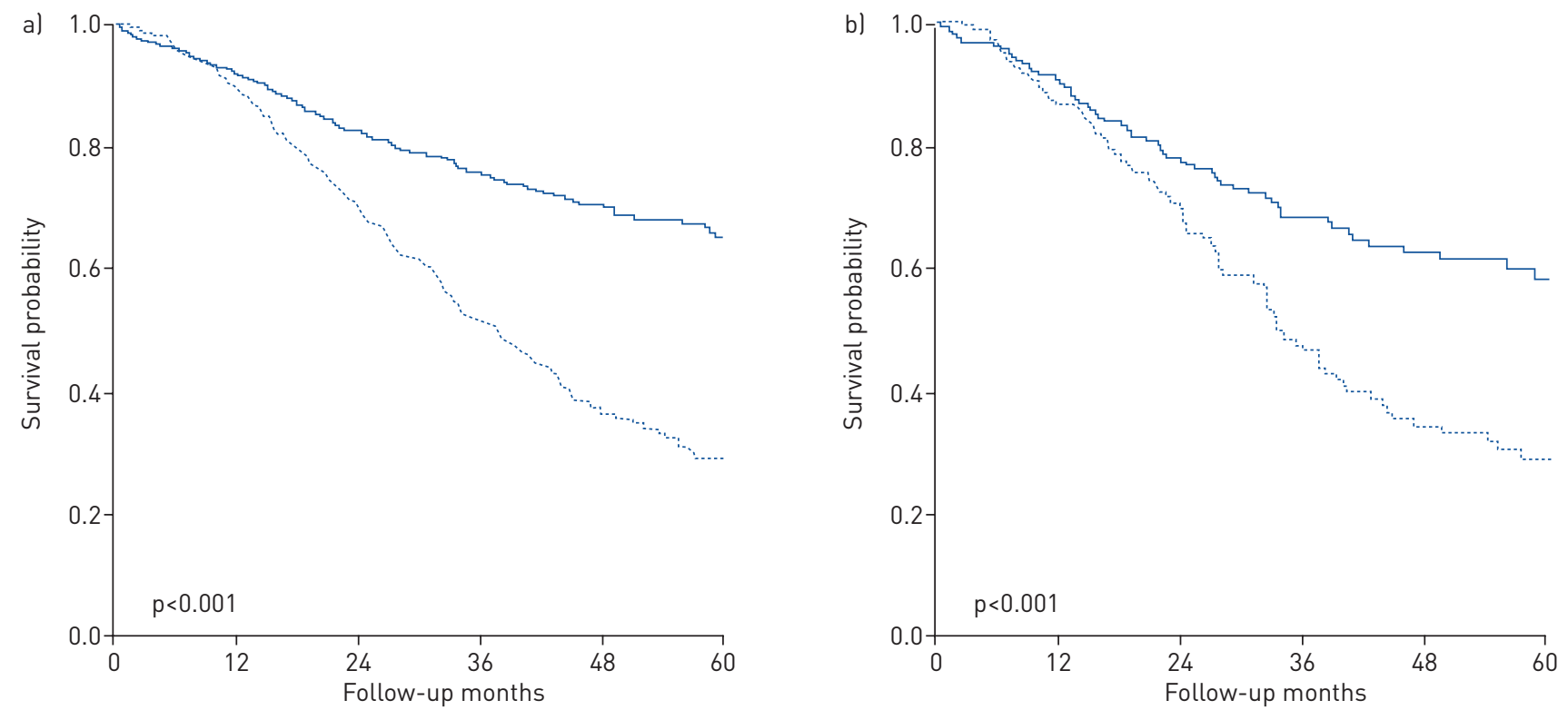

\begin{tabular}{lllllll}
\multicolumn{2}{l}{ No at risk } & & & & & \\
VATS & 414 & 381 & 342 & 225 & 145 & 85 \\
SBRT & 378 & 339 & 266 & 144 & 77 & 38
\end{tabular}

No at risk

VATS 159

SBRT 159

\section{4}

123

111

$\begin{array}{lll}77 & 54 & 33 \\ 52 & 29 & 14\end{array}$

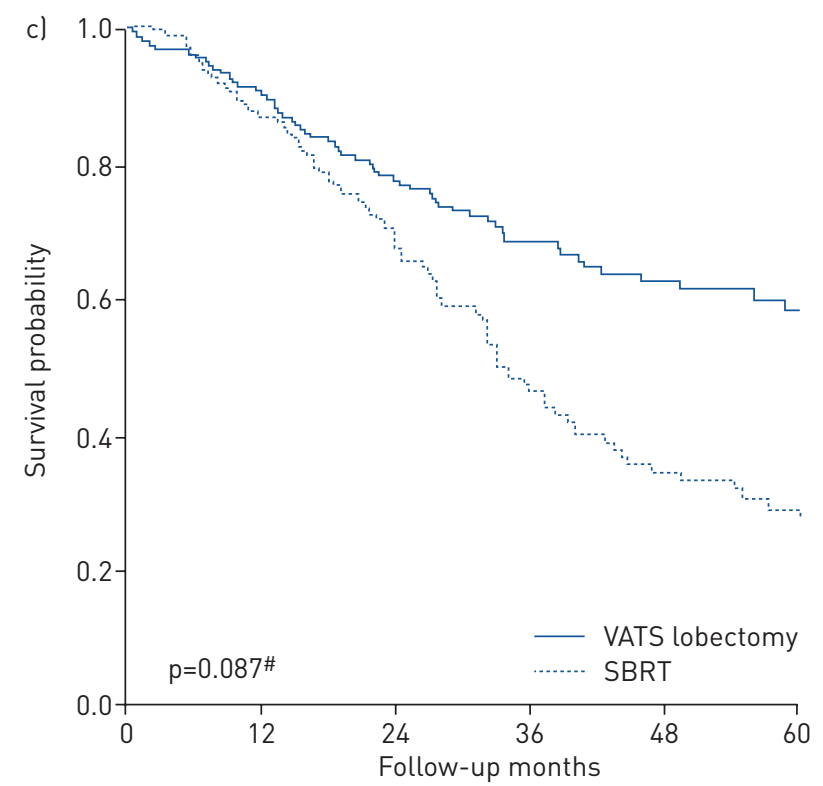

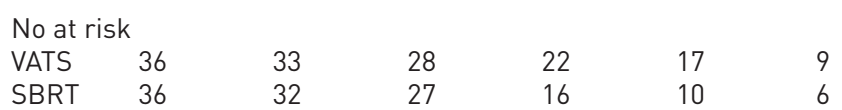

FIGURE 1 Kaplan-Meier curves for patients aged $\geqslant 65$ years undergoing video-assisted thoracic surgery (VATS) lobectomy and stereotactic body radiotherapy (SBRT) for clinical stage I nonsmall cell lung cancer in the full cohort (a), primary analysis (b) and secondary analysis (c). ${ }^{\#}$ : $p=0.034$ when adjusted for cT1a, histology and pathological confirmation.

with a number of previous studies comparing surgery with SBRT [14-16, 22], and studies specifically comparing VATS lobectomy with SBRT $[23,25]$.

Nevertheless, some studies reported no difference in survival between surgery and SBRT $[9,10,12,13,18]$, even in patients with operable disease [11, 20, 30, 31]. Indeed, LAGERWAARD et al. [20] reported 3- and 5 -year OS rates of $85 \%$ and $51 \%$, respectively, for patients with operable disease with stage I NSCLC treated with SBRT, which were similar to rates for surgery. A pooled analysis of the STARS and ROSEL trials suggests that both treatments are equally effective and the authors conclude that SBRT can be considered a treatment option in patients with operable disease who need a lobectomy [11]. A note of 
TABLE 3 Overall survival of patients aged $\geqslant 65$ years after intended VATS lobectomy and SBRT for clinical stage I nonsmall cell lung cancer for the first 15 months and after 15 months of follow-up

\begin{tabular}{|c|c|c|c|}
\hline & Subjects $\mathbf{n}$ & Hazard ratio $(95 \% \mathrm{CI})$ & p-value \\
\hline Full cohort & 792 & & \\
\hline First 15 months & & $0.66(0.44-0.99)$ & 0.044 \\
\hline After 15 months & & $0.33(0.26-0.43)$ & $<0.001$ \\
\hline Primary analysis & 318 & & \\
\hline First 15 months & & $0.87(0.49-1.55)$ & 0.64 \\
\hline After 15 months & & $0.38(0.26-0.56)$ & $<0.001$ \\
\hline Secondary analysis & 72 & & \\
\hline First 15 months & & $1.23(0.38-4.03)$ & 0.73 \\
\hline Adjusted $^{\#}$ & & $0.67(0.17-2.69)$ & 0.57 \\
\hline After 15 months & & $0.36(0.15-0.86)$ & 0.022 \\
\hline Adjusted ${ }^{\#}$ & & $0.30(0.11-0.81)$ & 0.018 \\
\hline
\end{tabular}

VATS: video-assisted thoracic surgery; SBRT: stereotactic body radiotherapy. " : adjusted for cT1a, histology and pathological confirmation because these variables were not well balanced after matching in the secondary analysis.

caution must be sounded because the STARS and ROSEL trials had different inclusion criteria, the analysis was underpowered due to a low number of patients and the trials had a short follow-up.

In both the primary and secondary analyses, there was no difference in OS between intended VATS lobectomy and SBRT in the first 15 months of follow-up. This could be explained by higher 30- and 90-day mortality after surgery compared to SBRT [32]. Mortality among surgical patients decreases after 90 days while it increases among patients undergoing SBRT. This might also explain why studies with a short follow-up do not find differences in OS between surgery and SBRT.

In the present study, intended VATS lobectomy patients with pathological stage I NSCLC had an excellent 5 -year OS rate of $70 \%$. Patients receiving SBRT had a significant survival disadvantage irrespective of matching. The higher OS after intended VATS lobectomy may be related to more complete tumour staging because a definite histological diagnosis is obtained. Pathological proof of malignancy was not obtained in $48 \%$ of patients receiving SBRT, which has been associated with inferior OS [3, 33]. Compromised pulmonary function and poor performance status may be reasons for deciding not to obtain pathological proof of malignancy and they are also associated with inferior OS [5, 34]. Higher OS after intended VATS lobectomy is probably not caused by selecting easy cases for VATS because almost two-thirds of surgically treated patients underwent VATS and one-third underwent thoracotomy between 2010 and 2015. After surgery, $22 \%$ of patients were upstaged: $52 \%$ of them received adjuvant treatment resulting in a 5 -year OS of $52 \%$ compared to $39 \%$ among those not receiving adjuvant treatment. The higher OS for patients undergoing intended VATS lobectomy might in part be explained by the increased survival following adjuvant treatment. Previous studies investigating the effect of adjuvant chemotherapy endorse this finding; an absolute increase in 5-year OS of $4 \%$ was reported in a meta-analysis by BURDETT et al. [35], and a 5-year OS gain of 15\% has also been described [36]. Adjuvant treatment could be offered earlier to patients undergoing SBRT once pretreatment staging has been improved to enable accurate identification of nodal disease.

There are potential biases influencing outcomes in many previous studies even after propensity score matching. Often no distinction is made between patients treated by thoracotomy or VATS [9-17]. All patients with clinical stage I NSCLC who are ineligible for surgery can undergo VATS, i.e. this is independent of pulmonary function or comorbidities. VATS mitigates the risk of post-operative morbidity and mortality [5,37], and could be useful in patients who cannot tolerate thoracotomy owing to compromised pulmonary function or multiple comorbidities [38, 39]. Many studies included mixed extents of resection $[10,13,16-18]$, which precludes comparison because the extent of resection is related to the prognosis of NSCLC [40]. In addition, elderly patients with multiple comorbidities often cannot tolerate resection more extensive than lobectomy $[5,14,22]$. We argue that studies comparing surgery with SBRT do not provide a reliable answer to the question of which treatment strategy will result in better OS. An ongoing randomised study, NCT02357992, assesses the effectiveness of SBRT in patients with operable disease. Other randomised studies, including NCT01753414 and NCT00499330, are underway to examine the effectiveness of surgery versus SBRT and different types of surgical resection. The present study could form the basis of future trials comparing one type of minimally invasive surgical resection with SBRT. 
TABLE 4 Characteristics of matched patients aged $\geqslant 65$ years undergoing intended VATS lobectomy and SBRT for clinical stage I nonsmall cell lung cancer

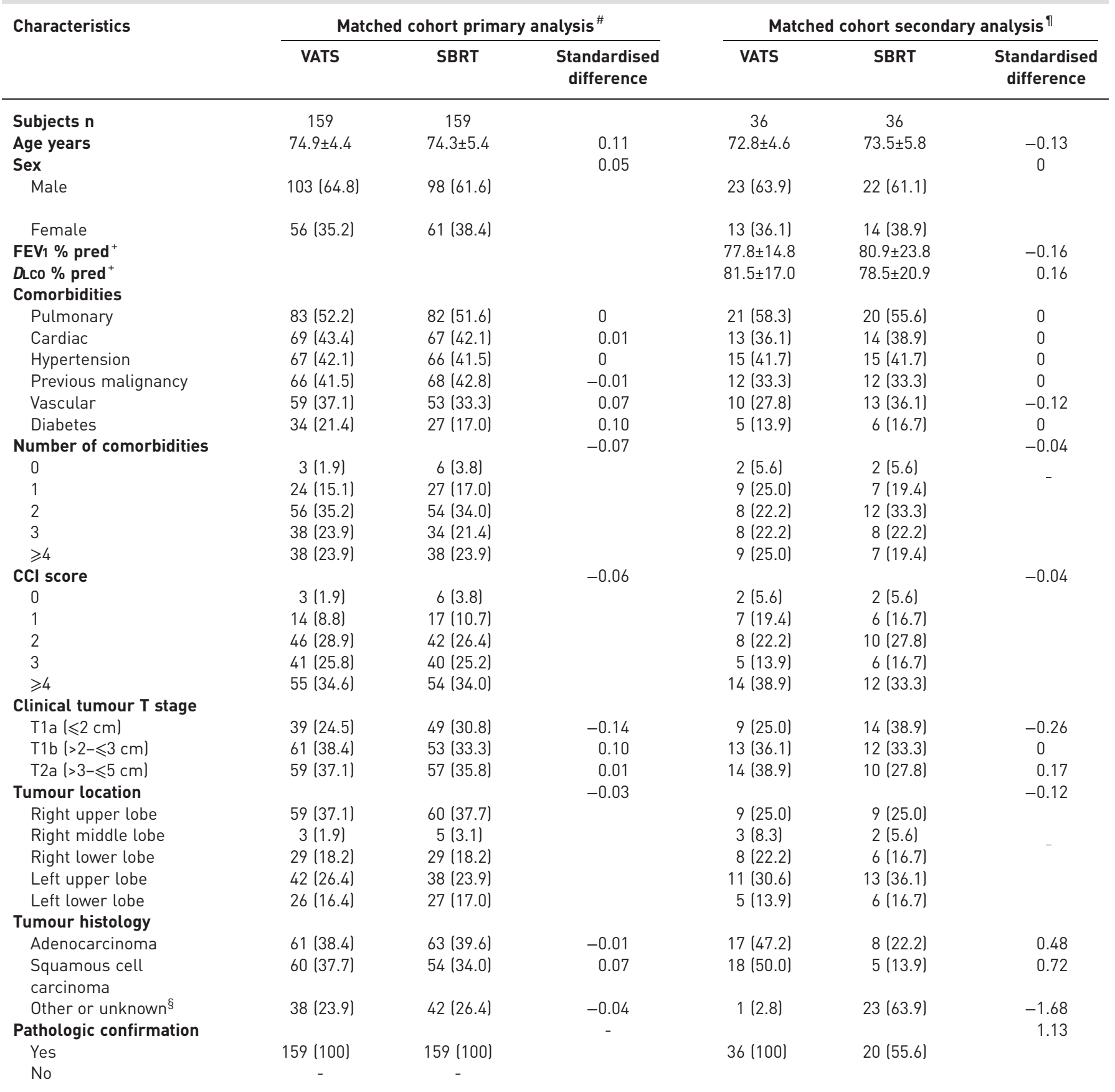

Data are presented as $\mathrm{n}(\%)$ or mean \pm SD, unless otherwise stated. VATS: video-assisted thoracic surgery; SBRT: stereotactic body radiotherapy; FEV1 \% pred: percentage predicted forced expiratory volume in $1 \mathrm{~s} ;$ LLCo \% pred: percentage predicted diffusing capacity of the lung for carbon monoxide; $\mathrm{CCl}$ : Charlson Comorbidity Index. ${ }^{\#}$ : matching on full cohort without data on pulmonary function; ": matching on subgroup with data on pulmonary function; ${ }^{+}$: variable used only in secondary analysis; ${ }^{\S}$ : the 16 patients without pathological confirmation undergoing SBRT in the secondary analysis are included in "other or unknown" tumour histology.

The results of this study must be seen in the context of its strengths and limitations. One of the strengths is the availability of information on comorbidities, which is often lacking or not used for matching in other studies $[10,13,16]$. Furthermore, the patient population truly reflected clinical practice; in many clinical trials the population consists of selected, relatively fit patients. Our results are therefore more generalisable than reports from single centres. In addition, clinical staging was used for both patients 
undergoing intended VATS lobectomy and patients undergoing SBRT. Analyses from the Surveillance, Epidemiology and End Results (SEER) database use clinical stage for patients undergoing SBRT and pathological staging for patients undergoing surgery, which limits the generalisability of the results [22]. Limitations of this study include its retrospective study design and all limitations associated with propensity score matching, which does not ensure that all SBRT patients are good surgical candidates, and cannot account for unmeasured or unselected confounders [17]. In addition, the 68 patients in whom a conversion was recorded were analysed as having undergone VATS lobectomy. This could have influenced the outcomes, although differences in patient characteristics remained the same when the 68 patients with a conversion were excluded. We did not exclude these patients because this truly reflects daily clinical practice. Whether VATS lobectomy provides better long-term survival than thoracotomy is unclear. A recent study reported better long-term survival after VATS lobectomy [41] while another recent study did not find a significant difference [42]. Disease-free survival and cancer-specific survival are not recorded in the NCR, although these are important parameters in the long-term follow-up of elderly patients with early-stage NSCLC. Furthermore, the absence of data on performance status and paucity of data on pulmonary function limits the present study. Incomplete data on pulmonary function excluded the majority of patients for matching, resulting in a small group of matched patients for the secondary analysis. Another reason for this small group of matched patients was that the groups were different by nature; e.g. there were significant differences in age and number of comorbidities in the unmatched patients. Despite this limitation, every effort was made to reduce imbalance. Matching was done on many covariates and the small standardised differences obtained suggested adequate balance, which minimises the confounding effect $[26,27]$. To account for the small but existing unbalance in the secondary analysis, we distinguished between matching on the selected variables with and without adjustment for the unbalanced variables. The caliper used eliminates at least $98 \%$ of the bias in the crude estimator [21]. Larger calipers used in other studies are likely to increase imbalance between subgroups, which favours surgery [17]. Despite matching as accurately as possible, selection bias was not completely nullified.

In conclusion, this analysis suggests that patients aged $\geqslant 65$ years with clinical stage I NSCLC undergoing intended VATS lobectomy might have better OS than patients undergoing SBRT, irrespective of matching. This could be clinically important in decision-making, especially in elderly patients who can tolerate surgical resection. Despite matching, there are limitations to this observational analysis and randomised trials remain necessary before widespread implementation of SBRT in the treatment of patients with operable NSCLC.

Acknowledgements: The authors thank all registrars that registered the patients in the database, as well as the Netherlands Cancer Registry. They thank Winfried W.C. Gieskes (University of Groningen, the Netherlands) for revising earlier versions of the manuscript.

Conflict of interest: None declared.

\section{References}

1 Global Burden of Disease Cancer Collaboration. Global, regional, and national cancer incidence, mortality, years of life lost, years lived with disability, and disability-adjusted life-years for 32 cancer groups, 1990 to 2015: a systematic analysis for the Global Burden of Disease Study. JAMA Oncol 2017; 3: 524-548.

2 Rijksinstituut voor volksgezondheid en milieu (RIVM). Future trend of new cases of lung cancer in the Net herlands. 2018. www.volksgezondheidenzorg.info/onderwerp/longkanker/cijfers-context/trends\#node-toekomst ige-trend-nieuwe-gevallen-longkanker. Date last accessed: October 8, 2018.

3 Detillon DDEMA, Driessen EJM, Aarts MJ, et al. Changes in treatment patterns and survival in elderly patients with stage I non-small-cell lung cancer with the introduction of stereotactic body radiotherapy and video-assisted thoracic surgery. Eur J Cancer 2018; 101: 30-37.

4 Bollen EC, Belgers E, van Haren EH, et al. De chirurgische behandeling van longkanker via complete VATSlobectomie, nieuw in Nederland [Surgical treatment of lung cancer with complete VATS lobectomy, new in the Netherlands]. Ned Tijdschr Geneeskd 2008; 152: 1204-1209.

5 Detillon DDEMA, Veen EJ. Postoperative outcome after pulmonary surgery for non-small cell lung cancer in elderly patients. Ann Thorac Surg 2018; 105: 287-293.

6 Haasbeek CJ, Palma D, Visser O, et al. Early-stage lung cancer in elderly patients: a population-based study of changes in treatment patterns and survival in the Netherlands. Ann Oncol 2012; 23: 2743-2747.

7 National Comprehensive Cancer Network Clinical Practice Guidelines: Non-small Cell Lung Cancer. 2015. www. oncoline.nl/niet-kleincellig-longcarcinoom. Date last accessed: October 12, 2018

8 Ball D, Mai T, Vinod S, et al. A randomized trial of SABR $v s$ conventional radiotherapy for inoperable stage $\mathrm{i}$ non-small cell lung cancer: TROG09.02 (CHISEL). J Thorac Oncol 2017; 12: Suppl. 2, S1853.

9 Mokhles S, Verstegen N, Maat AP, et al. Comparison of clinical outcome of stage I non-small cell lung cancer treated surgically or with stereotactic radiotherapy: results from propensity score analysis. Lung Cancer 2015; 87: 283-289.

10 Palma D, Visser O, Lagerwaard FJ, et al. Treatment of stage I NSCLC in elderly patients: a population-based matched-pair comparison of stereotactic radiotherapy versus surgery. Radiother Oncol 2011; 101: 240-244.

11 Chang JY, Senan S, Paul MA, et al. Stereotactic ablative radiotherapy versus lobectomy for operable stage I non-small-cell lung cancer: a pooled analysis of two randomised trials. Lancet Oncol 2015; 16: 630-637. 
12 Shirvani SM, Jiang J, Chang JY, et al. Lobectomy, sublobar resection, and stereotactic ablative radiotherapy for early-stage non-small cell lung cancers in the elderly. JAMA Surg 2014; 149: 1244-1253.

13 Kastelijn EA, El Sharouni SY, Hofman FN, et al. Clinical outcomes in early-stage NSCLC treated with stereotactic body radiotherapy versus surgical resection. Anticancer Res 2015; 35: 5607-5614.

14 Puri V, Crabtree TD, Bell JM, et al. Treatment outcomes in stage I lung cancer: a comparison of surgery and stereotactic body radiation therapy. J Thorac Oncol 2015; 10: 1776-1784.

15 Rosen JE, Salazar MC, Wang Z, et al. Lobectomy versus stereotactic body radiotherapy in healthy patients with stage I lung cancer. J Thorac Cardiovasc Surg 2016; 152: 44-54.

16 Robinson CG, DeWees TA, El Naqa IM, et al. Patterns of failure after stereotactic body radiation therapy or lobar resection for clinical stage I non-small-cell lung cancer. J Thorac Oncol 2013; 8: 192-201.

17 Chen H, Laba JM, Boldt RG, et al. Stereotactic ablative radiotherapy versus surgery in early lung cancer: a meta-analysis of propensity score studies. Int J Radiat Oncol Biol Phys 2018; 101: 186-194.

18 Puri V, Crabtree TD, Kymes S, et al. A comparison of surgical intervention and stereotactic body radiation therapy for stage I lung cancer in high-risk patients: a decision analysis. J Thorac Cardiovasc Surg 2012; 143: 428-436.

19 Union for International Cancer Control. TNM Classification of Malignant Tumours, 7th Edition. Chichester, Wiley-Blackwell, 2011.

2020 Lagerwaard FJ, Verstegen NE, Haasbeek CJ, et al. Outcomes of stereotactic ablative radiotherapy in patients with potentially operable stage I non-small cell lung cancer. Int J Radiat Oncol Biol Phys 2012; 83: 348-353.

21 Austin PC. Optimal caliper widths for propensity-score matching when estimating differences in means and differences in proportions in observational studies. Pharm Stat 2011; 10: 150-161.

22 Paul S, Lee PC, Mao J, et al. Long term survival with stereotactic ablative radiotherapy (SABR) versus thoracoscopic sublobar lung resection in elderly people: national population based study with propensity matched comparative analysis. BMJ 2016; 354: i3570.

23 Hamaji M, Chen F, Matsuo Y, et al. Video-assisted thoracoscopic lobectomy versus stereotactic radiotherapy for stage I lung cancer. Ann Thorac Surg 2015; 99: 1122-1129.

24 Verstegen NE, Oosterhuis JW, Palma DA, et al. Stage I-II non-small-cell lung cancer treated using either stereotactic ablative radiotherapy (SABR) or lobectomy by video-assisted thoracoscopic surgery (VATS): outcomes of a propensity score-matched analysis. Ann Oncol 2013; 24: 1543-1548.

25 Cornwell LD, Echeverria AE, Samuelian J, et al. Video-assisted thoracoscopic lobectomy is associated with greater recurrence-free survival than stereotactic body radiotherapy for clinical stage I lung cancer. J Thorac Cardiovasc Surg 2018; 155: 395-402.

26 Austin PC. Balance diagnostics for comparing the distribution of baseline covariates between treatment groups in propensity-score matched samples. Stat Med 2009; 28: 3083-3107.

27 Austin PC. Using the standardized difference to compare the prevalence of a binary variable between two groups in observational research. Commun Stat Simul Comput 2009; 38: 1228-1234.

28 Cohen J. Statistical Power Analysis for the Behavioral Sciences. 2nd Edn. Hillsdale, NJ, Lawrence Erlbaum Associates, 1988.

29 Austin PC. A tutorial and case study in propensity score analysis: an application to estimating the effect of in-hospital smoking cessation counseling on mortality. Multivariate Behav Res 2011; 46: 119-151.

30 Eriguchi T, Takeda A, Sanuki N, et al. Stereotactic body radiotherapy for operable early-stage non-small cell lung cancer. Lung Cancer 2017; 109: 62-67.

31 Onishi H, Shirato H, Nagata Y, et al. Stereotactic body radiotherapy (SBRT) for operable stage I non-small-cell lung cancer: can SBRT be comparable to surgery? Int J Radiat Oncol Biol Phys 2011; 81: 1352-1358.

32 Stokes WA, Bronsert MR, Meguid RA, et al. Post-treatment mortality after surgery and stereotactic body radiotherapy for early-stage non-small-cell lung cancer. J Clin Oncol 2018; 36: 642-651.

33 Palma D, Visser O, Lagerwaard FJ, et al. Impact of introducing stereotactic lung radiotherapy for elderly patients with stage I non-small-cell lung cancer: a population-based time-trend analysis. J Clin Oncol 2010; 28: 5153-5159.

34 Alexander M, Wolfe R, Ball D, et al. Lung cancer prognostic index: a risk score to predict overall survival after the diagnosis of non-small-cell lung cancer. Br J Cancer 2017; 117: 744-751.

35 Burdett S, Pignon JP, Tierney J, et al. Adjuvant chemotherapy for resected early-stage non-small cell lung cancer. Cochrane Database Syst Rev 2015; 3: CD011430.

36 Winton T, Livingston R, Johnson D, et al. Vinorelbine plus cisplatin vs. observation in resected non-small-cell lung cancer. N Engl J Med 2005; 352: 2589-2597.

37 Desai H, Natt B, Kim S, et al. Decreased in-hospital mortality after lobectomy using video-assisted thoracoscopic surgery compared with open thoracotomy. Ann Am Thorac Soc 2017; 14: 262-266.

38 Ceppa DP, Kosinski AS, Berry MF, et al. Thoracoscopic lobectomy has increasing benefit in patients with poor pulmonary function: a Society of Thoracic Surgeons Database analysis. Ann Surg 2012; 256: 487-493.

39 Donahoe LL, de Valence M, Atenafu EG, et al. High risk for thoracotomy but not thoracoscopic lobectomy. Ann Thorac Surg 2017; 103: 1730-1735.

40 Deng HY, Wang YC, Ni PZ, et al. Radiotherapy, lobectomy or sublobar resection? A meta-analysis of the choices for treating stage I non-small-cell lung cancer. Eur J Cardiothorac Surg 2017; 51: 203-210.

41 Al-Ameri M, Bergman P, Franco-Cereceda A, et al. Video-assisted thoracoscopic versus open thoracotomy lobectomy: a Swedish nationwide cohort study. I Thorac Dis 2018; 10: 3499-3506.

42 Yang CJ, Kumar A, Klapper JA, et al. A national analysis of long-term survival following thoracoscopic versus open lobectomy for stage I non-small-cell lung cancer. Ann Surg 2019; 269: 163-171. 